\title{
The effect of indacaterol/glycopyrronium versus tiotropium or salmeterol/fluticasone on the prevention of clinically important deterioration in COPD
}

\author{
This article was published in the following Dove Press journal: \\ International Journal of COPD \\ 4 May 2017 \\ Number of times this article has been viewed
}

\author{
Antonio R Anzueto ${ }^{1,2}$ \\ Claus F Vogelmeier ${ }^{3}$ \\ Konstantinos Kostikas ${ }^{4}$ \\ Karen Mezzi ${ }^{4}$ \\ Sebastian Fucile ${ }^{5}$ \\ Giovanni Bader ${ }^{4}$ \\ Steven Shen ${ }^{5}$ \\ Donald Banerji ${ }^{5}$ \\ Robert Fogel ${ }^{5}$ \\ 'University of Texas Health Science \\ Center, ${ }^{2}$ South Texas Veterans \\ Healthcare System, University of \\ Texas, San Antonio, TX, United States; \\ ${ }^{3}$ Department of Medicine, Pulmonary \\ and Critical Care Medicine, University \\ Medical Center Giessen and Marburg, \\ Philipps-Universität Marburg, \\ Marburg, Germany; ${ }^{4}$ Novartis Pharma \\ AG, Basel, Switzerland; ${ }^{5}$ Novartis \\ Pharmaceuticals Corporation, East \\ Hanover, NJ, United States
}

\begin{abstract}
Background: Endpoints that evaluate deterioration rather than improvement of disease may have clinical utility in COPD. In this analysis, we compared the effects of different maintenance treatments on the prevention of clinically important deterioration (CID) in moderate-to-severe COPD patients.
\end{abstract}

Methods: Data were analyzed from three 26-week studies comparing indacaterol/ glycopyrronium (IND/GLY) with tiotropium (TIO) or salmeterol/fluticasone (SFC). Two definitions of CID were used; each was a composite of three outcome measures typically associated with COPD. Definition 1 (D1) comprised a $\geq 100 \mathrm{~mL}$ decrease in forced expiratory volume in 1 second $\left(\mathrm{FEV}_{1}\right), \mathrm{a} \geq 4$-unit increase in St George's Respiratory Questionnaire, and a moderate-to-severe COPD exacerbation. In Definition 2 (D2), a $\geq 1$-unit decrease in transition dyspnea index replaced $\mathrm{FEV}_{1}$.

Results: Using D1, IND/GLY significantly reduced the risk of first or sustained CID versus either TIO (hazard ratio 0.72 [0.61, 0.86], $P=0.0003$ and 0.73 [0.61, 0.89], $P=0.001$ ) or SFC (0.67 [0.57, 0.80] and 0.63 [0.52, 0.77], both $P<0.0001)$. With D2, IND/GLY significantly reduced the risk of first, but not sustained, CID versus TIO ( 0.80 [0.64 to 0.99 ], $P=0.0359$ and 0.85 [0.66, 1.10], $P=0.2208)$ and both first and sustained CID versus SFC $(0.73[0.61,0.88]$, $P=0.001$ and $0.72[0.58,0.90], P=0.0036)$.

Conclusion: These data confirm the utility of the CID endpoint as a means of monitoring COPD worsening in patients with moderate-to-severe COPD. Using the CID measure, we demonstrated that dual bronchodilation with IND/GLY significantly reduced the risk of CID versus either long-acting muscarinic antagonist or long-acting $\beta_{2}$-agonist/inhaled corticosteroid treatment, providing further evidence for the benefit of dual bronchodilation in this patient population.

Keywords: IND/GLY, deterioration, COPD

\section{Introduction}

COPD is a preventable and treatable condition, characterized by persistent airflow limitation that is not fully reversible. Exacerbations, exertional dyspnea, worsening of symptoms, and deterioration of health status each contribute to disease severity. ${ }^{1}$

The endpoints routinely used in COPD clinical trials tend to evaluate the effects of treatments on improving spirometric and/or clinical parameters. Many patients, however, do not improve, developing instead acute and/or sustained deterioration of their disease. Indeed, deterioration in lung function, health status, and exacerbation frequency are all predictors of COPD worsening and/or mortality. ${ }^{2-6}$ These, too, are
Correspondence: Robert Fogel Novartis Pharmaceuticals Corporation, I Health Plaza, East Hanover 07936, NJ, United States

Tel +l 8627784884

Email robert.fogel@novartis.com (c) (i) (c) 2017 Anzueto et al. This work is published and licensed by Dove Medical Press Limited. The full terms of this license are available at https://www.dovepress.com/terms.php cc. ${ }_{\mathrm{BY}} \mathrm{NC}$ and incorporate the Creative Commons Attribution - Non Commercial (unported, v3.0) License (http://creativecommons.org/licenses/by-nch/3.0/). By accessing the work you hereby accept the Terms. Non-commercial uses of the work are permitted without any further permission from Dove Medical Press Limited, provided the work is properly attributed. For permission for commercial use of this work, please see paragraphs 4.2 and 5 of our Terms (https://www.dovepress.com/terms.php). 
therefore important outcome measures as they reflect the impact of treatments on preventing disease progression, which in itself is a major goal in COPD management. ${ }^{1}$

Since COPD is a multidimensional disease, composite endpoints that evaluate the effect of treatments not only on lung function but on COPD symptoms and exacerbations, as well as on general health status and quality of life, may be more sensitive to the effects of therapeutic interventions than individual measures, enabling a more comprehensive view of the overall disease. A recent publication described the development and use of one such endpoint in COPD patients. Termed clinically important deterioration (CID), it was used to assess, as a composite measure, the rate of deterioration in lung function, exacerbation rate, and health status following treatment with different classes of COPD medication. ${ }^{7}$ Using the CID endpoint, the authors demonstrated that the dual bronchodilator, umeclidinium/vilanterol (UMEC/VI) reduced the risk of first or sustained CIDs versus either placebo or bronchodilator monotherapy.

Dual bronchodilation with fixed-dose long-acting $\beta_{2}-$ agonist (LABA)/long-acting muscarinic antagonist (LAMA) combinations are often used as treatment options in patients with high symptom burden, since they prove more effective than current standard of care therapy with single-agent LAMAs or LABA/inhaled corticosteroid (ICS) combinations. ${ }^{8-10}$ Treatment differences between dual bronchodilators and either a single LABA or LAMA or a LABA/ICS, however, are often smaller than the differences seen versus placebo, so using a composite endpoint may provide a more sensitive means with which to compare active treatments.

The LABA/LAMA combination indacaterol/glycopyrronium (IND/GLY) has demonstrated significant improvement in lung function, dyspnea, quality of life, and exacerbation rates versus both the LAMA tiotropium (TIO) and the LABA/ ICS salmeterol/fluticasone (SFC) in COPD patients. ${ }^{8,10-12}$

In this analysis of studies from the IND/GLY (IGNITE) program, we describe the effect of IND/GLY versus both TIO and SFC on the risk of CID in COPD using patient data from three large randomized clinical trials. Our objective was to examine whether the benefits of IND/GLY observed in terms of improvement in COPD outcomes were also evident with regard to prevention of disease deterioration.

\section{Materials and methods Study design and population}

Data from three large multicenter, Phase 3 randomized studies, each part of the IGNITE program, were used in this analysis; the data analyzed were freely available as trial data points and/or published articles. Each study was of 26 weeks' duration and was conducted in patients with moderateto-severe COPD. SHINE (NCT01202188) ${ }^{8}$ compared the efficacy and safety of the dual bronchodilator IND/GLY with LAMA or LABA single bronchodilator therapy, while LANTERN (NCT01709903) ${ }^{11}$ and ILLUMINATE (NCT01315249) $)^{12}$ each compared IND/GLY combination therapy with the LABA/ICS combination SFC.

In SHINE, patients were randomized $(2: 2: 2: 2: 1)$ to receive IND/GLY 110/50 $\mu$ g once daily (o.d.), IND $150 \mu \mathrm{g}$ o.d., GLY $50 \mu$ g o.d., open-label TIO $18 \mu$ g o.d. or placebo. In LANTERN and ILLUMINATE, patients were randomized (1:1) to IND/GLY $110 / 50 \mu$ g o.d. or to SFC 50/500 $\mu \mathrm{g}$ twice daily. In order to improve the statistical power of our analysis, and as the populations were similar and used the same comparator, a combined data set of the LANTERN/ ILLUMINATE populations was used in the analysis.

\section{Patients}

\section{Key inclusion criteria}

In SHINE, men and women aged $\geq 40$ years were eligible to participate if they had received a diagnosis of moderate-tovery severe COPD according to Global Initiative for Chronic Obstructive Lung Disease guidelines, ${ }^{13}$ a post-bronchodilator forced expiratory volume in 1 second $\left(\mathrm{FEV}_{1}\right)$ of $\geq 30 \%$ to $<80 \%$ of predicted normal, ${ }^{13}$ and a post-bronchodilator $\mathrm{FEV}_{1}$ to forced vital capacity (FVC) ratio of $<0.7$.

In LANTERN/ILLUMINATE, patients aged $\geq 40$ years who were current or former smokers with a smoking history of at least 10 pack-years, and who had a post-bronchodilator $\mathrm{FEV}_{1}$ between $40 \%$ and $80 \%$ of predicted ${ }^{14}$ or $30 \%$ to $80 \%$ of predicted (LANTERN), ${ }^{15}$ and a post-bronchodilator $\mathrm{FEV}_{1}$ to $\mathrm{FVC}$ ratio of $<0.70$ were eligible.

\section{Key exclusion criteria}

In SHINE, patients with COPD exacerbations who required treatment with antibiotics, systemic corticosteroids (oral or intravenous), or hospitalization within 6 weeks before or during screening, or who had a respiratory tract infection within 4 weeks of screening were excluded from participation.

In LANTERN/ILLUMINATE, patients with any exacerbation (ILLUMINATE) or $\geq 2$ COPD exacerbations (LANTERN) who required treatment with antibiotics and/or oral corticosteroids and/or hospitalization in the year prior to screening or during the run-in period, were excluded.

\section{Definitions and assessment of first CID and sustained CID}

The risk of the first CID and a sustained CID were assessed using two definitions. These were based on the inverse of 
the minimal clinically important differences (MCIDs) for improvement of each of the composite variables employed (Table 1).

For Definition 1 (D1) we used the CID previously described by Singh et al. ${ }^{7}$ In this, a first CID was defined as any one of the following: $a \geq 100 \mathrm{~mL}$ decrease from baseline in trough $\mathrm{FEV}_{1}$; a $\geq 4$ unit increase in the St George's Respiratory Questionnaire (SGRQ) total score relative to the baseline measurement; or a moderate-to-severe COPD exacerbation that occurred after the first dose of study medication. A sustained CID using D1 was defined as $\mathrm{a} \geq 100 \mathrm{~mL}$ decrease from baseline in trough $\mathrm{FEV}_{1}$ on two consecutive visits at least 4 weeks apart or on $>50 \%$ of all subsequent visits; a $\geq 4$-unit increase in SGRQ total score from baseline on two consecutive visits at least 4 weeks apart or on $>50 \%$ of all subsequent visits; or a moderateto-severe COPD exacerbation occurring after the first dose of study medication.

In Definition 2 (D2) of a first CID, the FEV lung function component of D1 was replaced with a component measuring breathlessness: the transition dyspnea index (TDI). The definition of a CID for this component was also defined as a reversal of its widely used MCID; hence, a CID was defined as a $\geq 1$-unit deterioration in TDI from baseline, and this was considered a sustained CID if it was present on two consecutive visits at least 4 weeks apart, or on $>50 \%$ of all subsequent visits. The criteria used for SGRQ and exacerbations in D2 were the same as described for D1.

Time to a CID event, which was also documented in the analysis, was defined as the first time point at which the CID occurred.

\section{Statistical analyses}

All analyses were performed on the full analysis set, defined as patients who were randomized and received at least one dose of study treatment. Descriptive statistics (number and percentage) were used to summarize CID events. Median times with 95\% confidence intervals (CIs), as well as the $25 \%$ and $75 \%$ quintiles of the time-to-event are presented using Product Limit (Kaplan-Meier) plots.

Statistical comparisons of IND/GLY versus TIO (SHINE) and IND/GLY versus SFC (LANTERN/ILLUMINATE combined data set) were conducted for CID and sustained CID for both D1 and D2 definitions.

Kaplan-Meier curves for time-to-event data were generated for both data sets to illustrate patients' times to CIDs or sustained CIDs, and hazard ratios (HRs) with 95\% CIs, and $P$-values for treatment comparisons were analyzed using the Cox proportional hazard model. Covariates included in the model were treatment group, gender, age group, baseline COPD severity, ex-smoker (yes/no), and eosinophil count at baseline ( $>300$ or $\leq 300$ cells $/ \mu \mathrm{L})$. In addition, log rank tests were used to compare the KaplanMeier curves for treatment comparisons, with $P$-values presented alongside Kaplan-Meier curves. For time to CID and sustained CID analyses, patients without an event who remained on treatment were censored at the study end date; those who had discontinued were censored at their last study contact date.

Subgroup analyses were performed for each of the endpoints to explore the consistency of the overall treatment effect on the times to first and sustained CID. The subgroups used were based on gender, age ( $<65$ versus $\geq 65$ years), baseline COPD severity (moderate, severe, or very severe), smoking status (ex-smokers versus current smokers), and baseline blood eosinophil count ( $<300$ versus $\geq 300$ cells $/ \mu \mathrm{L}$ ).

In order to verify the main analysis results and to test the validity of using a composite endpoint, the time to first or sustained CID for each of the separate components of the composite endpoint were examined (these data are described in Figures $\mathrm{S} 1$ to $\mathrm{S} 3$ ).

Table I Definitions of first and sustained CID

\begin{tabular}{ll}
\hline Definition Ia & Definition $\mathbf{2}$ \\
\hline First CID (any of the following): & First CID (any of the following): \\
$\geq 100 \mathrm{~mL} \downarrow$ in trough FEV, from baseline & $\geq I$ unit $\downarrow$ in TDI score from baseline \\
$\geq 4$ unit $\uparrow$ in SGRQ score from baseline & $\geq 4$ unit $\uparrow$ in SGRQ score from baseline \\
An on-treatment moderate-to-severe COPD exacerbation & An on-treatment moderate-to-severe COPD exacerbation \\
Sustained CID (any of the following): & Sustained CID (any of the following): \\
$\geq 100 \mathrm{~mL} \downarrow$ in trough FEV, from baseline on two consecutive & $\geq I$ unit $\downarrow$ in TDI score from baseline on two consecutive visits \\
visits at least 4 weeks apart, or on $>50 \%$ of all subsequent visits & at least 4 weeks apart, or on $>50 \%$ of all subsequent visits \\
$\geq 4$ unit $\uparrow$ in SGRQ from baseline on two consecutive visits at & $\geq 4$ unit $\uparrow$ in SGRQ from baseline on two consecutive visits \\
least 4 weeks apart, or on $>50 \%$ of all subsequent visits & at least 4 weeks apart, or on $>50 \%$ of all subsequent visits \\
On-treatment moderate-to-severe COPD exacerbation & On-treatment moderate-to-severe COPD exacerbation \\
\hline
\end{tabular}

Note: as described by Singh et al, $2016 .{ }^{7}$

Abbreviations: CID, clinically important deterioration; FEV , forced expiratory volume in I second; SGRQ, St George's Respiratory Questionnaire; TDI, transition dyspnea index. 


\section{Results}

\section{Study populations}

The analysis populations comprised 954 patients for SHINE and 1,263 patients for the pooled LANTERN/ILLUMINATE study populations. Demographics and baseline characteristics were generally similar across treatment groups in both populations (Table 2). In SHINE, the majority of patients had moderate $\mathrm{COPD}^{8}$ while in LANTERN/ILLUMINATE the majority were male, ex-smokers who had experienced no exacerbations in the previous 12 months. ${ }^{11,12}$

\section{Incidence and time to first and sustained CID using DI of the composite CID endpoint \\ SHINE}

The incidence of a first CID was comparatively lower in the IND/GLY group compared with the TIO group ( $47 \%$ versus 59\%, respectively). Using D1, IND/GLY significantly reduced the risk of both first and sustained CID compared with TIO (HR 0.72 [0.61, 0.86], $P=0.0003$ and 0.73 [0.61, 0.89 ], $P=0.0013$, respectively) (Figure $1 \mathrm{~A}$ and B). IND/ GLY relative to TIO treatment delayed the time to first CID by 68 days (median times to CID: 117 and 185 days, respectively).

\section{LANTERN/ILLUMINATE}

The proportion of patients experiencing a CID was comparatively lower in the IND/GLY group compared with the SFC group (38\% versus 50\%, respectively). Per D1, IND/GLY significantly reduced the risk of both first and sustained CID compared with SFC $(0.67[0.57,0.80]$ and 0.63 [0.52, 0.77], both $P<0.0001$ ) (Figure $2 \mathrm{~A}$ and $\mathrm{B}$ ). The median time to first CID was not estimable for IND/ GLY-treated patients.

\section{Incidence and time to first and sustained CID using D2 of the composite CID endpoint \\ SHINE}

The incidence of a first CID was also comparatively lower in the IND/GLY group compared with the TIO group using D2 for the composite CID endpoint (first CID: 33\% versus $39 \%$ ). Using D2, IND/GLY significantly reduced the risk of first CID compared with TIO (0.80 [0.64 to 0.99], $P=0.0359)$ but not that of sustained CID $(0.85[0.66,1.10], P=0.2208)$ (Figure 3A and B). Median times to first CID were not estimable for either treatment group.

\section{LANTERN/ILLUMINATE}

Using D2, the incidence of a first CID was also comparatively lower in the IND/GLY group compared with the SFC group for both the first and sustained CIDs (first CID: $32 \%$ versus 40\%; sustained CID: $23 \%$ versus $30 \%$, respectively). With D2, IND/GLY also significantly reduced the risk of both first and sustained CIDs compared with SFC $(0.73$ [0.61, 0.88], $P=0.0010$ and $0.72[0.58,0.90], P=0.0036$, respectively)

Table 2 Patient demographics and baseline characteristics in SHINE and LANTERN/ILLUMINATE studies

\begin{tabular}{|c|c|c|c|c|}
\hline \multirow[t]{3}{*}{ Characteristic } & \multicolumn{2}{|l|}{ SHINE $^{8}$} & \multicolumn{2}{|c|}{ LANTERN/ILLUMINATE"I,I2 } \\
\hline & IND/GLY & TIO & IND/GLY & SFC \\
\hline & $\begin{array}{l}\text { I I 0/50 } \mu \text { g o.d. } \\
n=474\end{array}$ & $\begin{array}{l}18 \mu g \text { o.d. } \\
n=480\end{array}$ & $\begin{array}{l}\text { I I } 0 / 50 \mu g \text { o.d. } \\
n=630\end{array}$ & $\begin{array}{l}50 / 500 \mu g \text { b.i.d. } \\
n=633\end{array}$ \\
\hline Age, years & $64.0(8.9)$ & $63.5(8.7)$ & $64.1(8.0)$ & $64.5(7.8)$ \\
\hline Male, n (\%) & $362(76.4)$ & $360(75.0)$ & $522(82.9)$ & $520(82.1)$ \\
\hline Current smoker, n (\%) & $192(40.5)$ & $189(39.4)$ & $219(34.8)$ & $223(35.2)$ \\
\hline \multicolumn{5}{|l|}{ COPD severity, n (\%) } \\
\hline Mild & 0 & 0 & $2(0.32)$ & I $(0.16)$ \\
\hline Moderate & $313(66.0)$ & $296(61.7)$ & $399(63.33)$ & $407(64.30)$ \\
\hline Severe & $161(34.0)$ & $184(38.3)$ & $226(35.87)$ & $221(34.91)$ \\
\hline BDI & $6.45(0.1)$ & $6.46(0.1)$ & $6.5(0.08)$ & $6.5(0.08)$ \\
\hline ICS users at baseline, $\mathrm{n}(\%)$ & $268(56.5)$ & $282(58.8)$ & $291(46.2)$ & $298(47.1)$ \\
\hline Post-bronchodilator $\mathrm{FEV}_{1}, \%$ predicted & $55.7(\mid 3.2)$ & $55.1(13.5)$ & $55.2(12.7)$ & $55.3(12.6)$ \\
\hline \multicolumn{5}{|c|}{ Number of COPD exacerbations in previous year, $\mathrm{n}(\%)$} \\
\hline 0 & $352(74.3)$ & $363(75.6)$ & $569(90.3)$ & $539(85.2)$ \\
\hline I & $94(19.8)$ & $93(19.4)$ & $60(9.5)$ & $94(14.8)$ \\
\hline$\geq 2$ & $28(5.9)$ & $24(5.0)$ & $\mathrm{I}(0.16)$ & 0 \\
\hline
\end{tabular}

Note: Data presented as mean ( \pm SD), unless otherwise specified.

Abbreviations: BDI, Baseline Dyspnea Index; b.i.d., twice daily; FEV , forced expiratory volume in I second; ICS, inhaled corticosteroid; IND/GLY, indacaterol/glycopyrronium; o.d., once daily; TIO, tiotropium; SFC, salmeterol/fluticasone. 


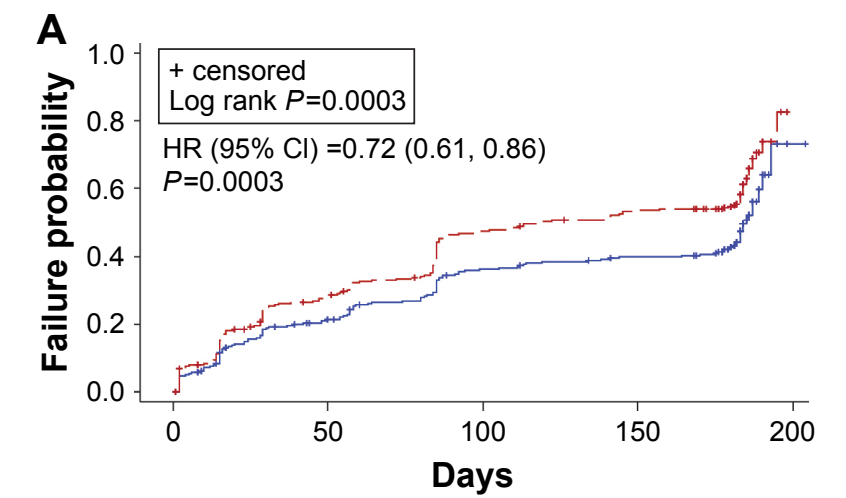

IND/GLY $474 \quad 363$

TIO $480 \quad 335$
B

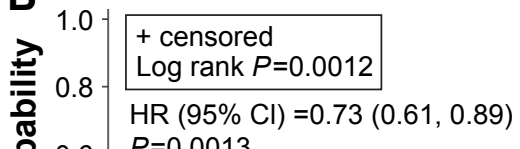

$P=0.0013$

$\begin{array}{lll}288 & 269 & 1 \\ 242 & 212 & 0\end{array}$

IND/GLY 474

TIO 480

\begin{tabular}{|l|}
\hline $\begin{array}{l}\text { Treatment } \\
\text { IND/GLY }\end{array} \quad---$ TIO \\
\hline
\end{tabular}

Figure I Kaplan-Meier curves and HR $(95 \% \mathrm{Cl})$ of time to first CID $(\mathbf{A})$ and sustained CID $(\mathbf{B})$ by DI (SHINE study ${ }^{8}$ ).

Abbreviations: HR, hazard ratio; Cl, confidence interval; CID, clinically important deterioration; DI, Definition I; IND/GLY, indacaterol/glycopyrronium; TIO, tiotropium.

(Figure 4A and B). Median times to first CID were not estimable for either treatment group.

\section{Subgroup analyses}

Subgroup analysis results were consistent with the overall results and are presented as forest plots in Figures 5 and 6. Hence, in patients treated with IND/GLY in SHINE, the risk of first and sustained CID was lower than in those treated with TIO across all subgroups, except for females in D1 (Figure 5A and B). The data followed a similar trend when D2 for CID was used, although CIs were larger (Figure 5C and D).
In LANTERN/ILLUMINATE, using either definition of CID, IND/GLY reduced the risk of first and sustained CID in all subgroups except those with high baseline eosinophil counts, where the high degree of data variability observed may have accounted for the variable results obtained (Figure 6).

\section{Discussion}

Our analysis demonstrated that in patients with moderateto-severe COPD, the dual bronchodilator IND/GLY significantly reduced the risk of a first CID versus either a
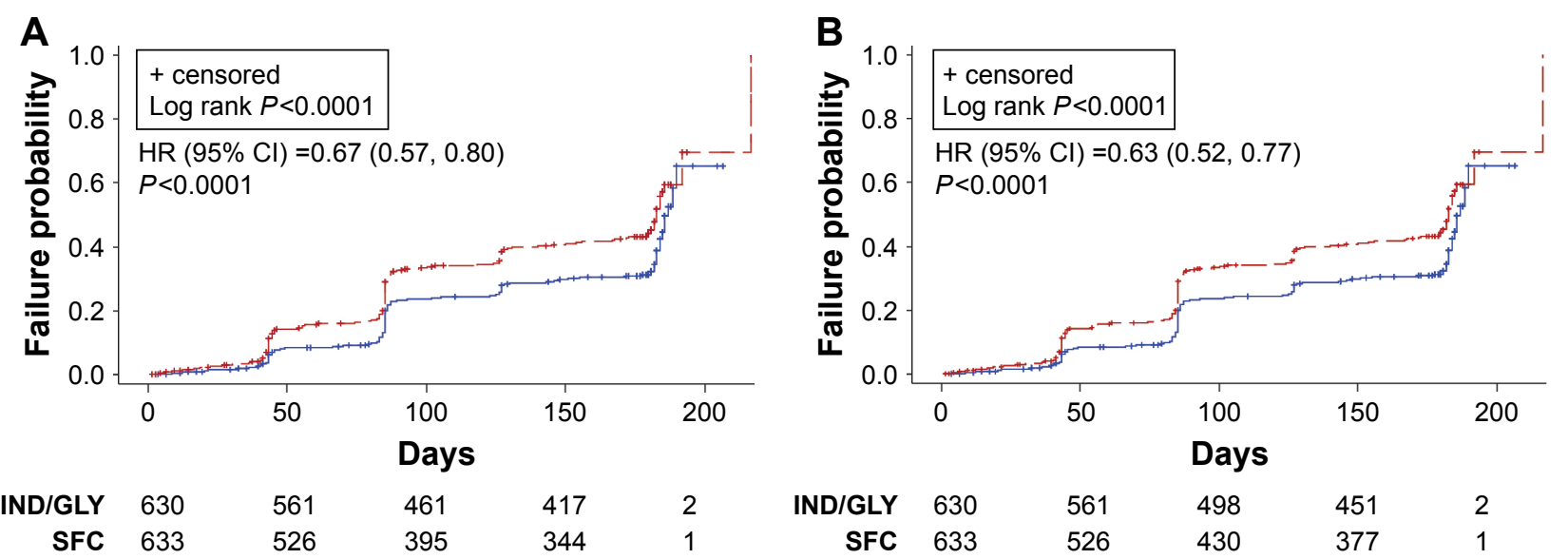

\begin{tabular}{|l|}
\hline Treatment \\
IND/GLY $\quad---$ SFC
\end{tabular}

Figure 2 Kaplan-Meier curves and HR $(95 \% \mathrm{Cl})$ of time to first CID $(\mathbf{A})$ and sustained CID $(\mathbf{B})$ by DI (LANTERN/ILLUMINATE $\left.{ }^{11,12}\right)$.

Abbreviations: HR, hazard ratio; Cl, confidence interval; CID, clinically important deterioration; DI, Definition I; IND/GLY, indacaterol/glycopyrronium; SFC, salmeterol/ fluticasone. 


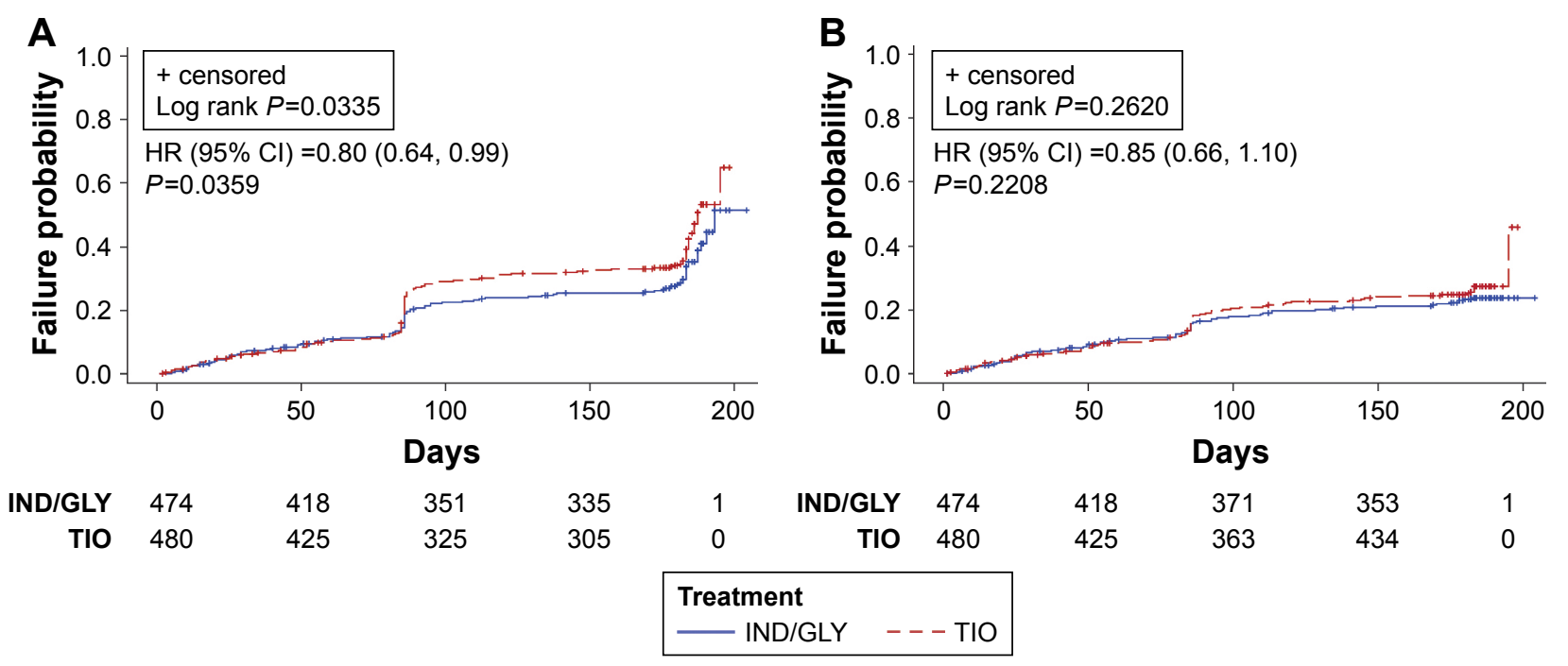

Figure 3 Kaplan-Meier curves and HR $(95 \% \mathrm{Cl})$ of time to first CID $(\mathbf{A})$ and sustained CID $(\mathbf{B})$ by D2 (SHINE study $\left.{ }^{8}\right)$.

Abbreviations: $\mathrm{HR}$, hazard ratio; $\mathrm{Cl}$, confidence interval; $\mathrm{CID}$, clinically important deterioration; D2, Definition 2; IND/GLY, indacaterol/glycopyrronium; TIO, tiotropium.

single-agent LAMA (TIO) or a LABA/ICS (SFC); this effect was seen with both definitions of CID. Sustained CIDs were also observed versus both comparators using D1, and versus SFC but not TIO using D2.

Subgroup analyses largely confirmed our findings. Apart from an apparent gender discrepancy versus TIO, and relatively high data variability in the subgroup of patients with higher baseline eosinophil count, the findings across the subgroups were generally consistent with the overall results, showing benefits in favor of IND/GLY versus either comparator.

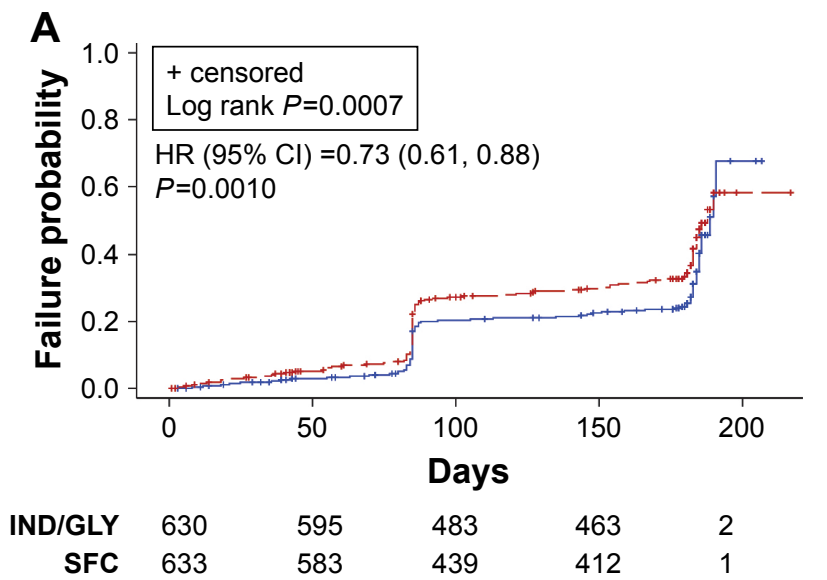

Composite measures in the form of prognostic indexes have long been used in COPD to categorize patients and predict survival. The BODE index, a multidimensional tool incorporating body mass index, degree of airflow obstruction, functional dyspnea, and exercise capacity was first described by Celli et al in 2004. ${ }^{16}$ They hypothesized that this composite endpoint would be better able to predict COPDrelated hospitalization and mortality than its individual components or the customary endpoint of $\mathrm{FEV}_{1}$ measurements alone. ${ }^{16}$ Since then, several other COPD indices have been described, which predict not only survival but disease-related



Figure 4 Kaplan-Meier curves and HR $(95 \% \mathrm{Cl})$ of time to first CID $(\mathbf{A})$ and sustained CID $(\mathbf{B})$ by D2 (LANTERN/ILLUMINATE $\left.{ }^{11,12}\right)$.

Abbreviations: HR, hazard ratio; Cl, confidence interval; CID, clinically important deterioration; D2, Definition 2; IND/GLY, indacaterol/glycopyrronium; SFC, salmeterol/ fluticasone. 
A

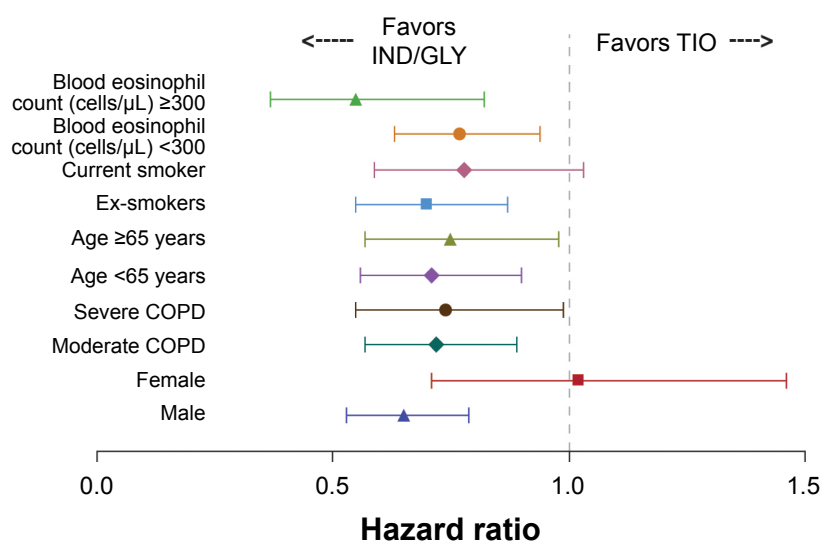

Hazard ratio

C

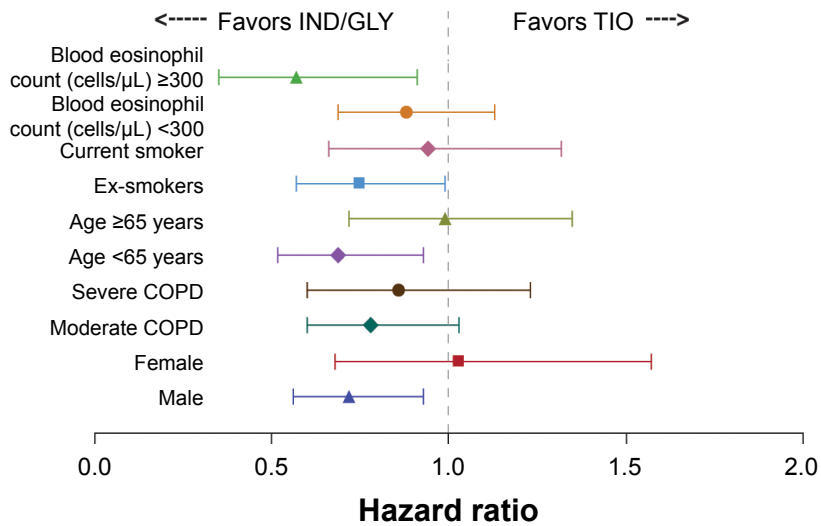

B

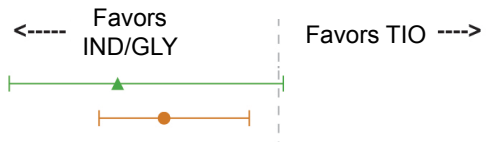

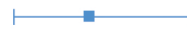
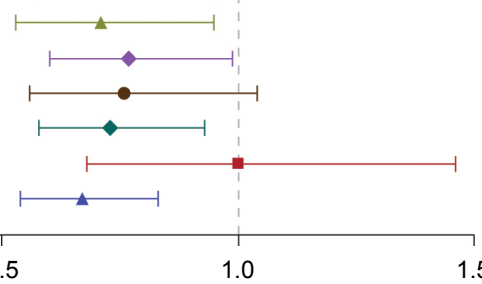

Hazard ratio

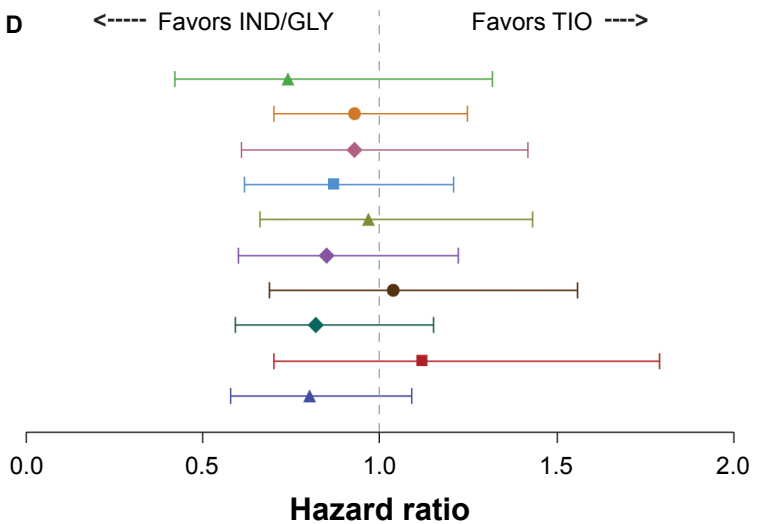

Figure 5 HRs and 95\% Cl (IND/GLY versus TIO) for first and sustained CID by subgroups in SHINE using DI (A and B) and D2 (C and D). Abbreviations: HR, hazard ratio; Cl, confidence interval; CID, clinically important deterioration; DI, Definition I; D2, Definition 2; IND/GLY, indacaterol/glycopyrronium; $\mathrm{TIO}$, tiotropium.

hospitalization or exacerbations, disease severity, and even positive outcomes in clinical trials. ${ }^{17-19}$ What distinguishes the composite endpoint of CID from these indices is that it can be used to compare different active treatments since it measures the relative deterioration in disease following an intervention, and as such could have utility in clinical trials as a means of monitoring disease worsening. As COPD is a usually progressive disease, some patients do not respond to treatment and for these patients treatments that prevent disease worsening and/or maintain stability would clearly be of value. Using an endpoint such as CID, which measures disease worsening rather than the usually analyzed improvement, would facilitate the identification of such treatments. Further validation of CID as an endpoint was shown in a recent post hoc analysis of data from two 3-year studies (TORCH and ECLIPSE), where long-term outcomes in terms of lung function, health status, exacerbation risk, and mortality were significantly worse in patients who had experienced an early CID (ie, within 6-12 months of follow-up). ${ }^{20}$ While composite indices have not been frequently used in respiratory trials, they have been standard measurements in many cardiovascular outcome studies where their value has been clearly demonstrated to differentiate between effective therapies. ${ }^{21,22}$ The data from this analysis, along with that of other analyses and from other therapeutic areas, suggest that a composite index may be more sensitive to detect treatment benefit, than a single measure such as $\mathrm{FEV}_{1}$. At the current time, however, most are likely too cumbersome to be routinely used in clinical practice.

When we examined time to first CID for the individual components of each CID definition, we observed that the strongest driver of CID in each of the analysis populations was lung function, with time to first $\mathrm{FEV}_{1} \mathrm{CID}$ event significantly longer in IND/GLY-treated patients versus TIO- or SFCtreated patients (both $P<0.0001$; Figures $\mathrm{S} 1$ and S2). This finding was not unexpected given that the treatments under study were bronchodilators whose most prominent effects are those related to lung function. Nevertheless, each of the other individual component measures also appeared to influence the risk of CID, albeit with relatively more variability. 

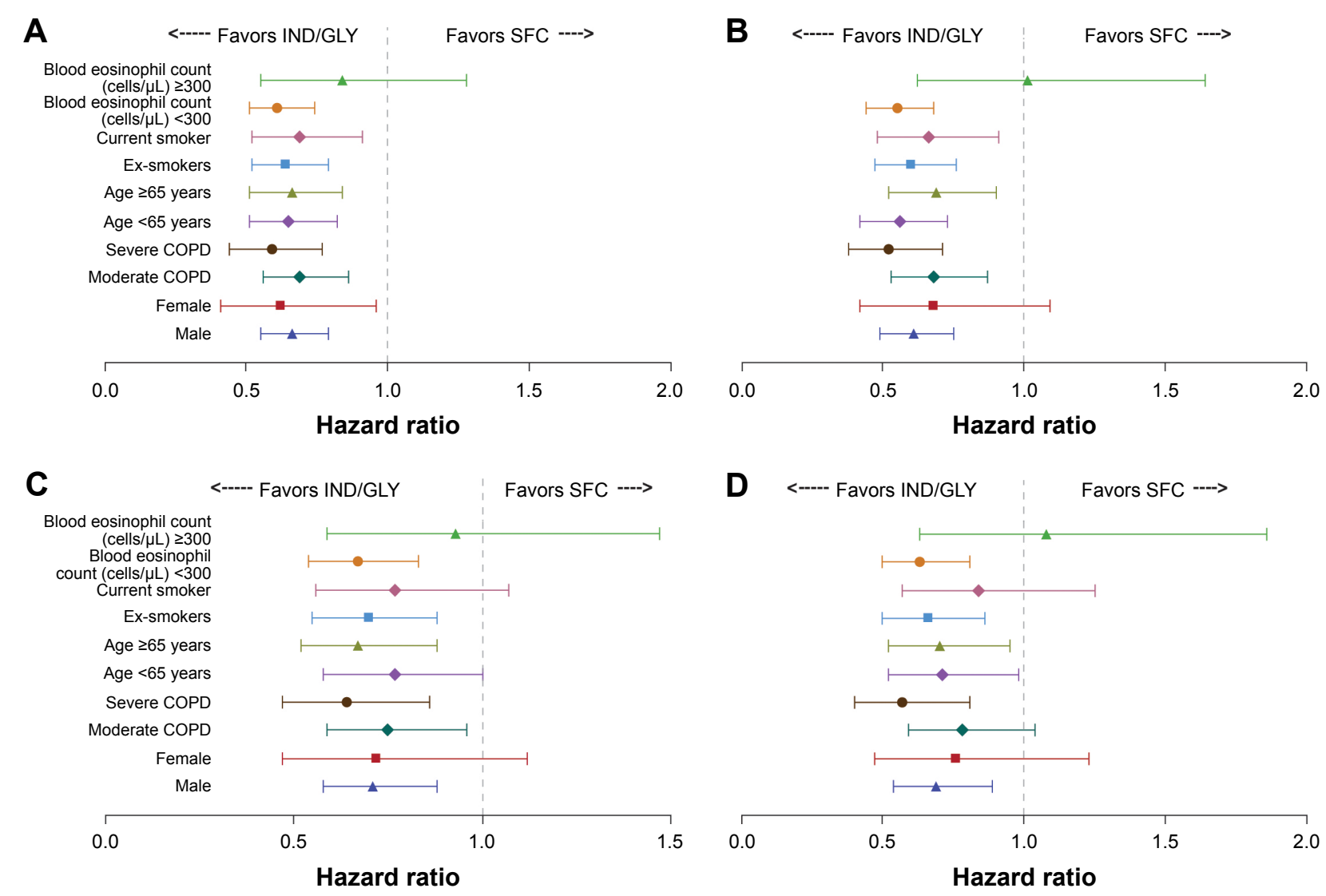

Figure 6 HRs and 95\% Cl (IND/GLY versus SFC) for first and sustained CID by subgroups in LANTERN/ILLUMINATE ${ }^{11,12}$ using DI (A and B) and D2 (C and D). Abbreviations: $\mathrm{HR}$, hazard ratio; $\mathrm{Cl}$, confidence interval; CID, clinically important deterioration; DI, Definition I; D2, Definition 2; IND/GLY, indacaterol/glycopyrronium; SFC, salmeterol/fluticasone.

In our analysis, we included two definitions of CID in order to provide a degree of sensitivity and some validation of CID as an endpoint. D1 has been described and used previously, ${ }^{7}$ showing that UMEC/VI reduced the risk of CID versus either placebo or bronchodilator monotherapy. ${ }^{7,23}$ For D2, we replaced FEV 1 with TDI, and did so to have a definition of CID composed solely of "clinical" endpoints rather than lung function. This definition appeared to be less sensitive than D1, and there was greater data variability, as evidenced by the larger CIs. This was not surprising, however, as TDI and SGRQ were measured relatively infrequently during the studies compared with $\mathrm{FEV}_{1}$, and this likely contributed to the smaller between-treatment differences observed for these endpoints. The latter probably also accounted for the variability observed in the results of sustained CID as, by definition, sustained CID would require more frequent measurement. Nevertheless, it is unclear why IND/GLY, using D2, significantly improved sustained CID versus SFC in LANTERN/ILLUMINATE but not versus TIO in the SHINE study, particularly since baseline dyspnea was similar in both populations. Prior exacerbations were slightly higher in SHINE than in LANTERN/ILLUMINATE, but were low in both study populations. However, while background ICS use was allowed to be continued in SHINE, it was discontinued in LANTERN/ILLUMINATE. It is possible, therefore, that such differences in background treatments may have affected a composite index designed to assess strictly clinical variables.

We recognize that there are limitations to our analysis. First, this analysis used data from clinical trials of relatively short duration (26 weeks) that had not been designed to assess treatment deterioration. Second, the exacerbation frequency of patients in our studies may have been relatively low, so our findings may not be applicable to patients at higher risk of exacerbation and the analysis should be repeated in patients at higher risk. We also acknowledge that currently there is no universally accepted definition of CID, and the thresholds we used to define a CID event were based on the inverse of the MCIDs of the component endpoints. Whether these are valid thresholds, in which positive or negative clinical benefit 
can be measured using the same increment of change, is not known and needs to be investigated in larger prospective trials; indeed, it may be the case that the threshold signifying a CID may vary with disease severity.

\section{Conclusion}

In conclusion, our data support the use of the CID endpoint as a means of monitoring disease stability versus worsening in patients with moderate-to-severe COPD. Prevention of disease deterioration is of obvious clinical benefit, and a measure that can differentiate between treatments in this regard has application to clinical practice. Using the CID endpoint, we have shown that dual bronchodilation with IND/ GLY offers significant benefits over treatment with a singleagent LAMA or a LABA/ICS both in terms of the incidence and time to CID, with some evidence of sustained efficacy. Therefore, these data support the use of dual bronchodilation in the studied patient population.

\section{Acknowledgments}

This analysis was sponsored by Novartis Pharma AG (Basel, Switzerland). Medical writing assistance was provided by Colette O'Sullivan, PhD, of Scriva Medical Communications Ltd, a professional medical writer funded by Novartis.

\section{Disclosure}

The authors take full responsibility for the scope, direction, content, and editorial decisions relating to the manuscript, and were involved at all stages of development and approved the submitted manuscript. The authors received no compensation related to the development of the manuscript.

ARA has acted as a consultant and served on advisory boards for Novartis Pharma AG, AstraZeneca, Boehringer Ingelheim, GSK, and Sunovion.

CFV has acted as a consultant and served on advisory boards for Almirall, AstraZeneca, Boehringer Ingelheim, Chiesi; received grants and personal fees from GlaxoSmithKline, grants and personal fees from Grifols, Mundipharma, Novartis, Takeda, Cipla, and Berlin Chemie/Menarini.

$\mathrm{KK}$ is an employee and shareholder of Novartis Pharma AG. Previously he has received honoraria for educational activities and lectures from AstraZeneca, Boehringer Ingelheim, Chiesi, ELPEN, GSK, Novartis, and Takeda, and has participated on advisory boards arranged by AstraZeneca, Chiesi, ELPEN, Novartis, and Takeda.

KM, SF, GB, SS, DB and RF are employees and shareholders of Novartis Pharma AG or Novartis Pharmaceuticals Corporation.
The authors have no other conflicts of interest to report in this work.

\section{References}

1. goldcopd.org [homepage on the Internet]. Global Strategy for the Diagnosis, Management and Prevention of COPD, Global Initiative for Chronic Obstructive Lung Disease (GOLD); as current in 2017. Available from: http://goldcopd.org. Accessed March 26, 2017.

2. Celli BR, Thomas NE, Anderson JA, et al. Effect of pharmacotherapy on rate of decline of lung function in chronic obstructive pulmonary disease: results from the TORCH study. Am J Respir Crit Care Med. 2008; 178(4):332-338.

3. Spencer S, Calverley PM, Sherwood Burge P, Jones PW; ISOLDE Study Group. Inhaled Steroids in Obstructive Lung Disease. Health status deterioration in patients with chronic obstructive pulmonary disease. Am J Respir Crit Care Med. 2001;163(1):122-128.

4. Wilke S, Jones PW, Mullerova H, et al. One-year change in health status and subsequent outcomes in COPD. Thorax. 2015;70(5):420-425.

5. Donaldson GC, Seemungal TA, Bhowmik A, Wedzicha JA. Relationship between exacerbation frequency and lung function decline in chronic obstructive pulmonary disease. Thorax. 2002;57(10):847-852.

6. Anzueto A. Impact of exacerbations on COPD. Eur Respir Rev. 2010;19(116):113-118.

7. Singh D, Maleki-Yazdi MR, Tombs L, Iqbal A, Fahy WA, Naya I. Prevention of clinically important deteriorations in COPD with umeclidinium/vilanterol. Int J Chron Obstruct Pulmon Dis. 2016;11: 1413-1424.

8. Bateman ED, Ferguson GT, Barnes N, et al. Dual bronchodilation with QVA149 versus single bronchodilator therapy: the SHINE study. Eur Respir J. 2013;42(6):1484-1494.

9. Wedzicha JA, Decramer M, Ficker JH, et al. Analysis of chronic obstructive pulmonary disease exacerbations with the dual bronchodilator QVA149 compared with glycopyrronium and tiotropium (SPARK): a randomised, double-blind, parallel-group study. Lancet Respir Med. 2013;1(3):199-209.

10. Wedzicha JA, Banerji D, Chapman KR, et al. IndacaterolGlycopyrronium versus Salmeterol-Fluticasone for COPD. $N$ Engl J Med. 2016;374(23):2222-2234.

11. Zhong N, Wang C, Zhou X, et al. LANTERN: a randomized study of QVA149 versus salmeterol/fluticasone combination in patients with COPD. Int J Chron Obstruct Pulmon Dis. 2015;10:1015-1026.

12. Vogelmeier CF, Bateman ED, Pallante J, et al. Efficacy and safety of once-daily QVA149 compared with twice-daily salmeterol-fluticasone in patients with chronic obstructive pulmonary disease (ILLUMINATE): a randomised, double-blind, parallel group study. Lancet Respir Med. 2013;1(1):51-60.

13. goldcopd.org [homepage on the Internet]. Global Strategy for the Diagnosis, Management and Prevention of COPD, Global Initiative for Chronic Obstructive Lung Disease (GOLD) guidelines; as current in 2008. Available from: http://goldcopd.org. Accessed March 26, 2017.

14. goldcopd.org [homepage on the Internet]. Global Strategy for the Diagnosis, Management and Prevention of COPD, Global Initiative for Chronic Obstructive Lung Disease (GOLD); as current in 2009. Available from: http://goldcopd.org. Accessed March 26, 2017.

15. goldcopd.org [homepage on the Internet]. Global Strategy for the Diagnosis, Management and Prevention of COPD, Global Initiative for Chronic Obstructive Lung Disease (GOLD); as current in 2010. Available from: http://goldcopd.org. Accessed March 26, 2017.

16. Celli BR, Cote CG, Marin JM, et al. The body-mass index, airflow obstruction, dyspnea, and exercise capacity index in chronic obstructive pulmonary disease. $N$ Engl J Med. 2004;350(10):1005-1012.

17. Briggs A, Spencer M, Wang H, Mannino D, Sin DD. Development and validation of a prognostic index for health outcomes in chronic obstructive pulmonary disease. Arch Intern Med. 2008; 168(1):71-79. 
18. Jones RC, Donaldson GC, Chavannes NH, et al. Derivation and validation of a composite index of severity in chronic obstructive pulmonary disease: the DOSE Index. Am J Respir Crit Care Med. 2009; 180(12):1189-1195.

19. Celli BR, Decramer M, Liu D, Metzdorf N, Asijee GM, Tashkin DP. Defining a COPD composite safety endpoint for demonstrating efficacy in clinical trials: results from the randomized, placebo-controlled UPLIFT $^{\circledR}$ trial. Respir Res. 2016;17(1):48.

20. Naya I, Tombs T, Mullerova H, Compton C, Jones P. Long-term outcome following first clinically important deterioration in COPD. Eur Respir J. 2016;48:304.

21. McMurray JJ, Packer M, Desai AS, et al. Angiotensin-neprilysin inhibition versus enalapril in heart failure. $N$ Engl J Med. 2014; 371(11):993-1004.
22. Tsutsui H, Momomura S, Saito Y, et al. Efficacy and safety of sacubitril/valsartan (LCZ696) in Japanese patients with chronic heart failure and reduced ejection fraction: Rationale for and design of the randomized, double-blind PARALLEL-HF study. J Cardiol. Epub 2016 Dec 23.

23. Maleki-Yazdi MR, Singh D, Anzueto A, Tombs L, Fahy WA, Naya I. Assessing short-term deterioration in maintenance-naive patients with COPD receiving umeclidinium/vilanterol and tiotropium: a pooled analysis of three randomized trials. Adv Ther. 2017; 33(12):2188-2199. 


\section{Supplementary materials Component analyses}

In order to verify the main analysis results and to test the validity of using a composite endpoint, the time to first or sustained clinically important deterioration (CID) for each of the separate components of the composite endpoint were examined.

Kaplan-Meier curves for time-to-event data were generated for both data sets to illustrate patients' times to CIDs or sustained CIDs, and hazard ratios with $95 \%$ confidence intervals, and $P$-values for treatment comparisons were analyzed using the Cox proportional hazard model. Covariates included in the model were treatment group, gender, age group, baseline COPD severity, ex-smoker (yes/no), and eosinophil count at baseline ( $>300$ or $\leq 300$ cells $/ \mu \mathrm{L})$. In addition, the log rank tests were used to compare the curves for treatment comparisons, and the $P$-values are presented alongside Kaplan-Meier curves. For time to CID and sustained CID analyses, patients without an event who remained on treatment were censored at the study end date; those who had discontinued were censored at their last study contact date.

The data are shown in Figures S1 and S2 for time to first event (ie, a CID of each separate component), and in Figure S3 for time to sustained CID. Apart from time to first exacerbation in $\mathrm{SHINE}^{1}$ and time to first transition dyspnea index deterioration in LANTERN/ILLUMINATE, ${ }^{2,3}$ indacaterol/ glycopyrronium (IND/GLY) versus either comparator significantly delayed the time to first CID for each of the separate components of the composite endpoint. The strongest driver of CID in each of the analysis populations was lung function, with time to first forced expiratory volume in 1 second CID
A Time to first exacerbation

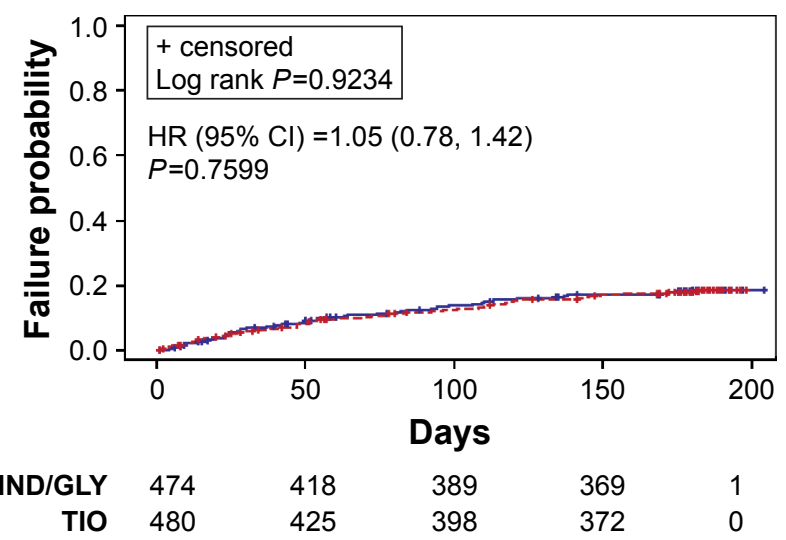

C

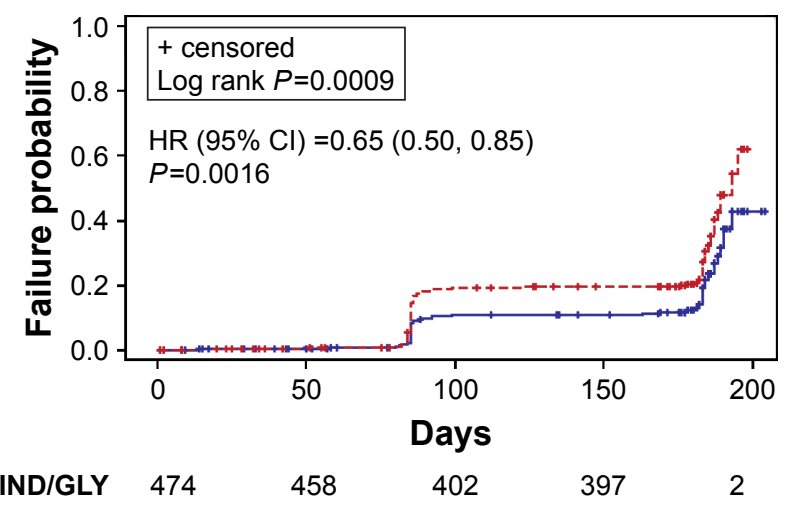

B

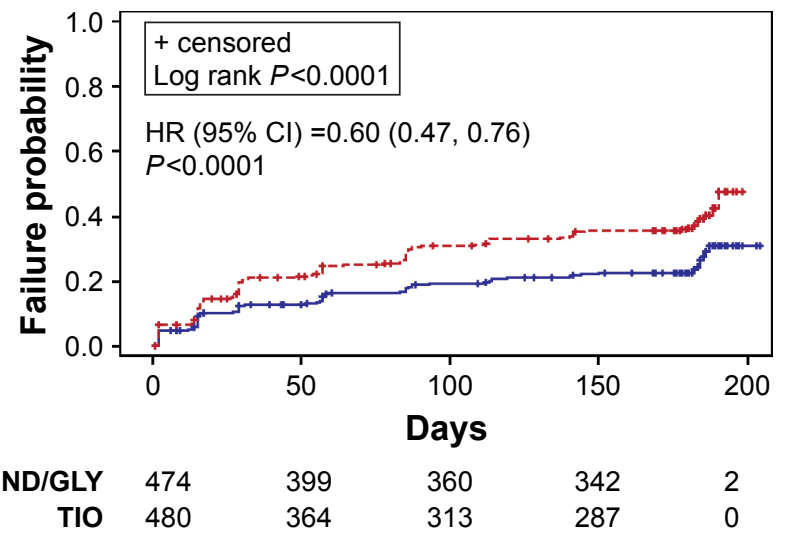

D

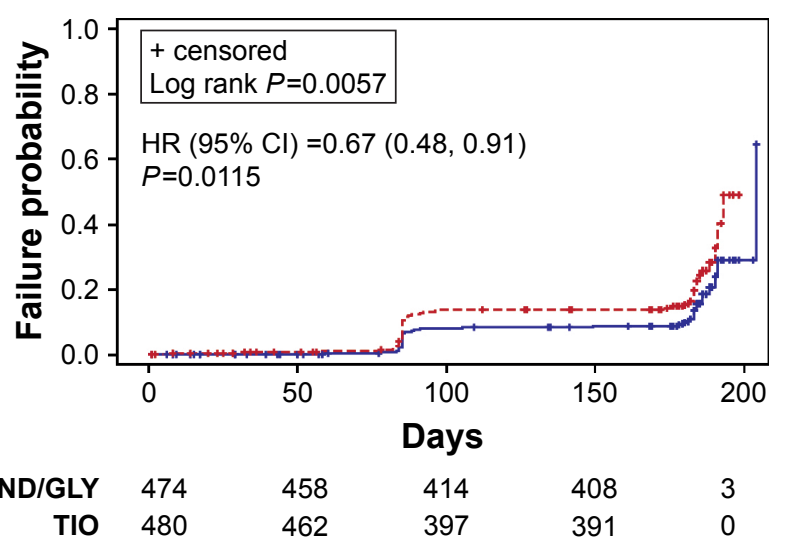

$\frac{\operatorname{trtp}}{\mathrm{IND} / \mathrm{GLY} \quad----\mathrm{TIO}}$

Figure SI Kaplan-Meier curves (with numbers of subjects at risk and $95 \% \mathrm{Cls}$ ) of time to first CID: IND/GLY versus TIO: SHINE': (A) time to first exacerbation, (B) time to first FEV $\leq-100$, (C) time to first SGRQ $\geq 4$, (D) time to first TDI $\leq-1$.

Abbreviations: $\mathrm{Cl}$, confidence interval; HR, hazard ratio; CID, clinically important deterioration; IND/GLY, indacaterol/glycopyrronium; TDI, Transition Dyspnea Index; $\mathrm{TIO}$, tiotropium; $\mathrm{FEV}_{1}$, forced expiratory volume in I second; SGRQ, St George's Respiratory Questionnaire; trtp, treatment. 
event significantly longer in IND/GLY-treated patients versus tiotropium (TIO)- or salmeterol/fluticasone (SFC)-treated patients (both $P<0.0001$; Figures S1 and S2, respectively). IND/GLY also significantly delayed the time to sustained
CID versus $\mathrm{TIO}$ in the SHINE population (Figure S3A). A numerical benefit versus SFC was observed in LANTERN/ ILLUMINATE, although this did not translate into statistical significance (Figure S3B).
A

Time to first exacerbation

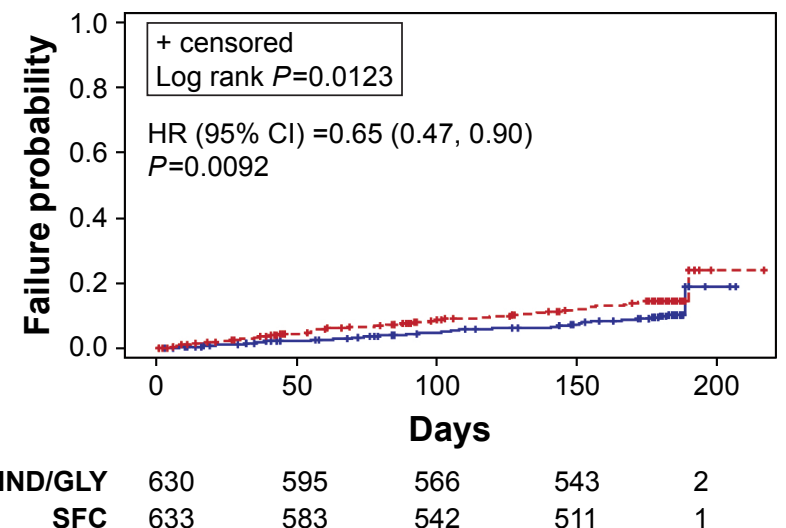

C

Time to first SGRQ $\geq 4$

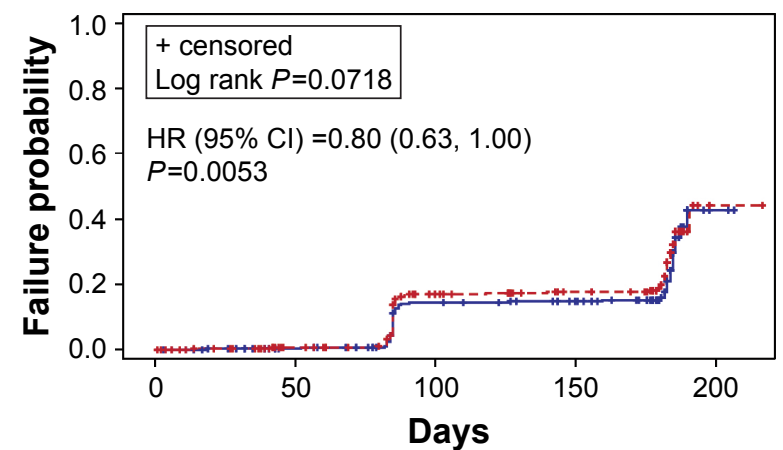

IND/GLY $630 \quad 608$

SFC $633 \quad 607$
513

501
467
B Time to first FEV $1 \leq-100$

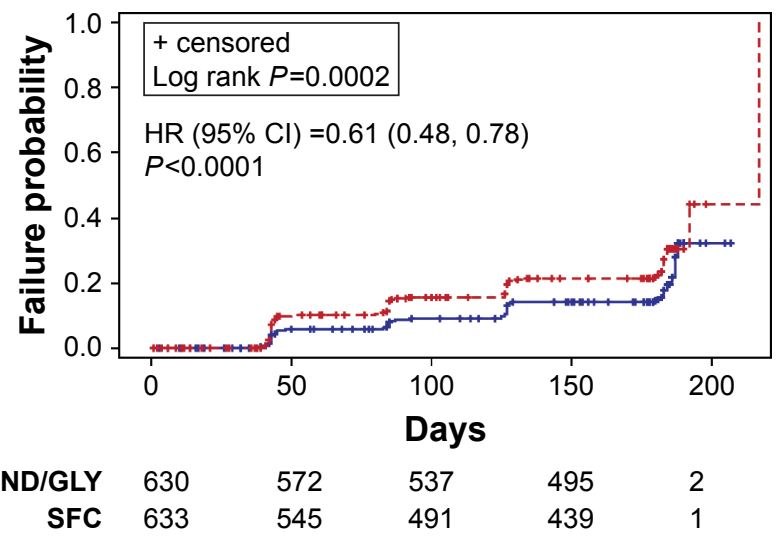

D $\quad$ Time to first TDI $\leq-1$

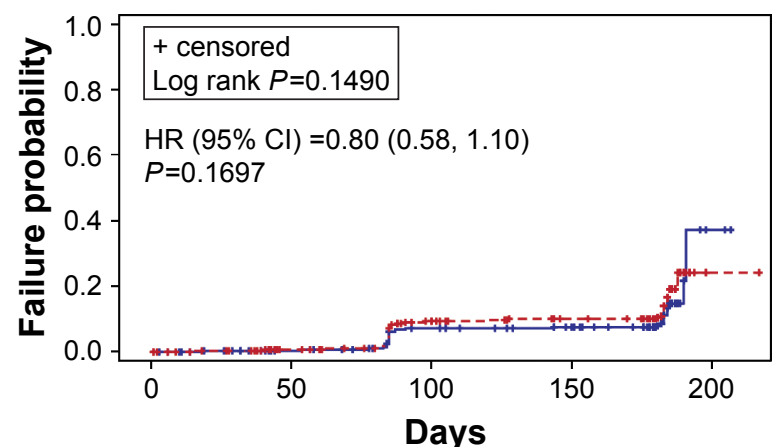

$\begin{array}{lllll}\text { IND/GLY } & 630 & 608 & 554 & 543\end{array}$ $\begin{array}{lllll}\text { SFC } & 633 & 606 & 538 & 521\end{array}$

exacerbation, (B) time to first $F E V, \leq-100,(\mathbf{C})$ time to first $S G R Q \geq 4$, (D) time to first TDI $\leq-1$.

Abbreviations: $\mathrm{Cl}$, confidence interval; HR, hazard ratio; CID, clinically important deterioration; IND/GLY, indacaterol/glycopyrronium; SFC, salmeterol/fluticasone; SGRQ, St George's Respiratory Questionnaire; TDI, Transition Dyspnea Index; trtp, treatment. 
A

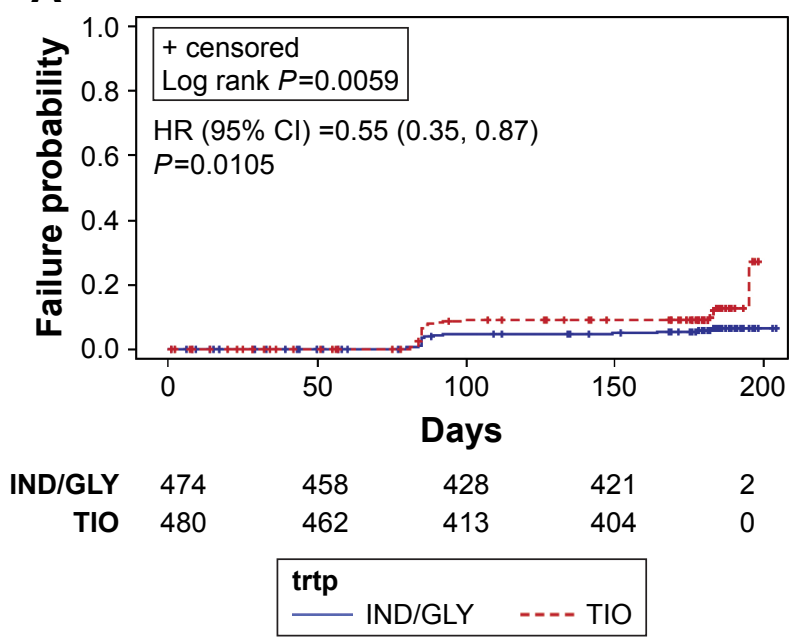

B

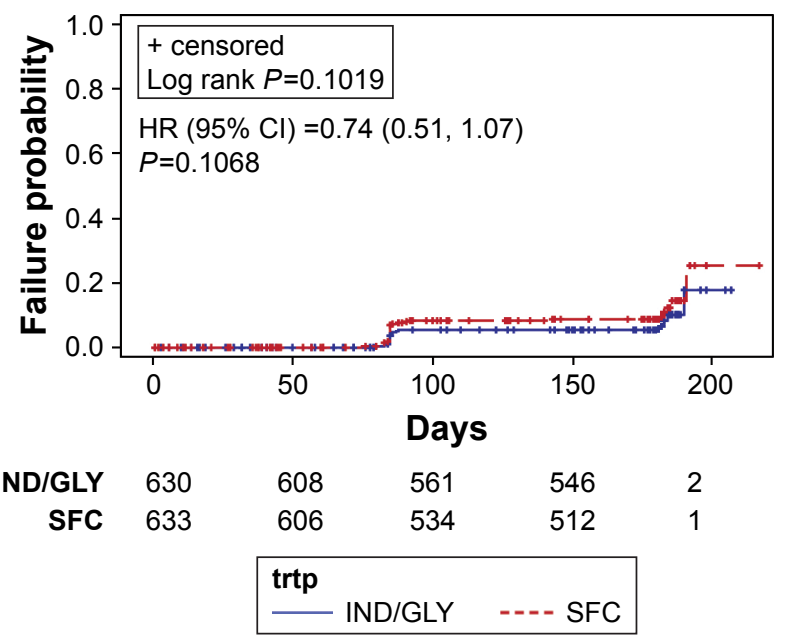

Figure S3 Kaplan-Meier curves (with numbers of subjects at risk and 95\% Cls) on the component of sustained CID for (A) IND/GLY versus TIO (SHINE') and (B) IND/GLY versus SFC (LANTERN/ILLUMINATE $\left.{ }^{2,3}\right)$.

Abbreviations: Cl, confidence interval; HR, hazard ratio; CID, clinically important deterioration; IND/GLY, indacaterol/glycopyrronium; TIO, tiotropium; SFC, salmeterol/ fluticasone; trtp, treatment.

\section{References}

1. Bateman ED, Ferguson GT, Barnes N, et al. Dual bronchodilation with QVA149 versus single bronchodilator therapy: the SHINE study. Eur Respir J. 2013;42(6):1484-1494.

2. Zhong N, Wang C, Zhou X, et al. LANTERN: a randomized study of QVA149 versus salmeterol/fluticasone combination in patients with COPD. Int J Chron Obstruct Pulmon Dis. 2015;10:1015-1026.
3. Vogelmeier CF, Bateman ED, Pallante J, et al. Efficacy and safety of once-daily QVA149 compared with twice-daily salmeterol-fluticasone in patients with chronic obstructive pulmonary disease (ILLUMINATE): a randomised, double-blind, parallel group study. Lancet Respir Med. 2013;1(1):51-60.
International Journal of COPD

\section{Publish your work in this journal}

The International Journal of COPD is an international, peer-reviewed journal of therapeutics and pharmacology focusing on concise rapid reporting of clinical studies and reviews in COPD. Special focus is given to the pathophysiological processes underlying the disease, intervention programs, patient focused education, and self management protocols.

\section{Dovepress}

This journal is indexed on PubMed Central, MedLine and CAS. The manuscript management system is completely online and includes a very quick and fair peer-review system, which is all easy to use. Visit $\mathrm{http}: / / \mathrm{www}$.dovepress.com/testimonials.php to read real quotes from published authors. 